\title{
Relato de caso: diagnóstico mascarado pela neuropatia diabética em um caso de hanseníase
}

\author{
Case report: masked diagnosis for diabetic neuropathy in leprosy case
}

Relato de caso: diagnóstico enmascarado por la neuropatía diabética en un caso de lepra

Caroline Gabriele Betiati Demito ${ }^{1 *}$, Isadora Dias Lacerda ${ }^{1}$, Isabela Rodrigues de Oliveira ${ }^{1}$, Wesley Lobo Costa Júnior ${ }^{1}$, Talitha Araújo Faria'.

\section{RESUMO}

Objetivo: Relatar o caso de um paciente adulto, sexo masculino, portador de diabetes mellitus e acometido por hanseníase. Detalhamento do caso: No caso relatado o paciente é diabético em tratamento irregular e sem acompanhamento médico periódico, o que propiciou o desenvolvimento de um quadro característico de consequente complicação da doença, o "pé diabético", sobreposto às sequelas neurológicas da hanseníase. A hanseníase é causada pelo bacilo $M$. leprae, que possui tropismo por células da pele e nervos periféricos, causando lesões cutâneas indolores. Considerações finais: Até o presente momento da elaboração do artigo, o paciente segue em tratamento poliquimioterápico para hanseníase, controle glicêmico rigoroso e reabilitação de sequelas.

Palavras chave: Diabetes mellitus, Hanseníase, Parestesia.

\begin{abstract}
Objective: To report the case of an adult patient, male, with diabetes mellitus and affected by leprosy. Case report: In the case reported, the patient is diabetic in irregular treatment and without periodic medical followup, which allowed the development of a characteristic picture of a consequent complication of the disease, the "diabetic foot", superimposed on the neurological sequelae of leprosy. Leprosy is caused by the M. leprae bacillus, which is tropism by skin cells and peripheral nerves, causing painless skin lesions. Final considerations: Until the present moment of the article's elaboration, the patient is undergoing polychemotherapy treatment for leprosy, rigorous glycemic control and rehabilitation.
\end{abstract}

Key words: Diabetes mellitus, Leprosy, Paresthesia.

\section{RESUMEN}

Objetivo: Informar el caso de un paciente adulto, sexo masculino, portador de diabetes mellitus y acometido por lepra. Detalle del caso: En el caso relatado el paciente es diabético en tratamiento irregular y sin acompañamiento médico periódico, lo que propició el desarrollo de un cuadro característico de consecuente complicación de la enfermedad, el "pie diabético", superpuesto a las secuelas neurológicas de la lepra. La hanseniasis es causada por el bacilo M. leprae, que posee tropismo por células de la piel y nervios periféricos, causando lesiones cutáneas indoloras.. Consideraciones finales: Hasta el presente momento de la elaboración del artículo, el paciente sigue en tratamiento poliquimioterápico para hanseniasis, control glucémico riguroso y rehabilitación de secuelas.

Palabras clave: Diabetes Mellitus, La lepra, Parestesia.

${ }^{1}$ Universidade Atenas do Estado de Minas Gerais, Paracatu-MG. * E-mail: carolinegbdemito@hotmail.com 


\section{INTRODUÇÃO}

O diabetes mellitus (DM) é uma patologia caracterizada basicamente por um quadro de hiperglicemia decorrente da falta ou incapacidade da insulina exercer sua função, ou seja, de captar glicose sanguínea e permitir sua absorção pelas células do organismo. A hiperglicemia se manifesta por sintomas como polifagia, polidipsia, poliúria, perda de peso e em casos crônicos com retinopatia, disfunção e danos de diversos sistemas como o cardiovascular e nervoso. O DM tipo 2 é mais comum e é caracterizado por distúrbios da ação ou secreção de insulina, sendo que ainda não apresenta etiologia claramente esclarecida. Possui faixa etária de início variável, embora seja mais frequente após os 40 anos de idade. (GROSS JL, et al., 2002; BORTOLETTO MSS, et al., 2009).

Os efeitos principais do diabetes mellitus são hiperglicemia crônica relativa, com alterações no metabolismo dos carboidratos, lipídios e proteínas; e as complicações macrovasculares, microvasculares e neuropáticas. Disfunções neuropáticas são recorrentes e normalmente são concomitantemente associadas a complicações como o "pé diabético, o qual é caracterizado pela presença de pelo menos uma das seguintes alterações: neurológicas, ortopédicas, vasculares e infecciosas, que podem ocorrer no pé do paciente portador de diabetes. As lesões provenientes do quadro de hiperglicemia crônica geralmente complicam-se com uma infecção, podendo culminar em um quadro de amputação se não tratado precoce e adequadamente. Frequentemente, amputações estão associadas com o aparecimento de calos e feridas nas plantas dos pés como consequência do atrito e da pressão excessiva associada a perda de sensibilidade nesses locais (CAIAFA JS, et al., 2011; CUBAS MR, et al., 2017).

Problemas neuropáticos também causam sintomas como dor, sob forma de queimação e ardor, diminuição da sensibilidade, fraqueza nas pernas e pés, junto a uma ausência dos reflexos dos membros inferiores (BORTOLETTO MSS, et al., 2009).

As formas mais comuns do diabetes são o DM tipo 1 e DM tipo 2, que se diferem por termos "dependentes de insulina" ou "não dependentes de insulina" respectivamente. O DM tipo 1 se caracteriza por destruição das células beta do pâncreas, produtoras de insulina, por processo autoimune ou idiopático (causa desconhecida). Como consequência da destruição das células beta pancreáticas há uma deficiência absoluta de insulina, caracterizando então indivíduos "dependentes de insulina". A maior incidência desse tipo de diabetes se dá na faixa etária de 10 aos 14 anos, porém pode desencadear-se em qualquer idade (GROSS JL, et al., 2002).

O diagnóstico do diabetes mellitus fundamenta-se nas alterações da glicose plasmática de jejum $(<126$ $\mathrm{mg} / \mathrm{dL}$; hemoglobina glicada (> 6,5\%); após sobrecarga de glicose via oral (> $200 \mathrm{mg} / \mathrm{dL}$ ) ou glicose ao acaso $>200 \mathrm{mg} / \mathrm{dL}$ com sintomas inequívocos de hiperglicemia (OLIVEIRA JEP, et al., 2017).

Embora não haja relações específicas entre diabetes e o quadro de hanseníase, sabe-se que o diabetes mellitus pode propiciar ou agravar infecções (LASTORIA JC, et al., 2006). A perda de sensibilidade característica do diabetes pode ser confundido e prejudicar o diagnóstico diferencial de hanseníase, que também se associa a perda de sensibilidade. A grande e essencial diferença é que o diabetes não faz surgir 
feridas e manchas, somente agrava-as devido as neuropatias; porém, a hanseníase possui esse grande fator clínico (SOUZA CFD, et al., 2010).

A hanseníase é uma doença infecciosa crônica cujo agente etiológico é o Mycobacterium leprae, bacilo intracelular obrigatório. Devido a preferência do bacilo por células da pele e nervos periféricos, clinicamente os pacientes apresentam lesões cutâneas múltiplas, difusas e indolores. Pode ser classificada em dois polos estáveis e opostos: tuberculóide ou wirchowiana, porém atualmente, para facilitar o diagnóstico e tratamento, utiliza-se a classificação de acordo com a quantidade de bacilos: multibacilar (MB) ou paucibacilar (PB) (GROSSI MA, 2003; LASTORIA JC, et al., 2006; PEREIRA HLA, et al., 2006).

O diagnóstico baseia-se no encontro de lesões cutâneas típicas com perda de sensibilidade, espessamento de nervos periféricos e baciloscopia positiva, verificada através do índice baciloscópico (IB). Há ainda o índice morfológico (IM), utilizado para descrever o aspecto morfológico dos bacilos. A baciloscopia positiva classifica o caso como multibacilar, independentemente do número de lesões (LASTORIA JC, et al., 2006; PEREIRA HLA, et al., 2006).

O tratamento da hanseníase compreende: quimioterapia especifica, supressão dos surtos reacionais, prevenção de incapacidades físicas, reabilitação física e psicossocial. A poli quimioterapia (PQT) com rifampicina, dapsona e clofazimina revela-se muito eficaz (GROSSI MA, 2003; LASTORIA JC, et al., 2006).

Um dos sintomas da hanseníase que se assemelha ao diabetes mellitus é a neuropatia; nesse caso o comprometimento de nervos periféricos ocorre por meio do processo inflamatório causado por ação direta do bacilo ou por reações imunológicas do próprio organismo devido a presença do bacilo. Como resultado há alterações sensitivas e motoras, responsáveis pelas incapacidades e deformidades físicas (GROSSI MA, 2003; LASTORIA JC, et al., 2006; PEREIRA HLA, et al., 2006).

No relato de caso apresentado, o paciente foi admitido para tratamento de lesão ulcerativa característica de "pé diabético" e evoluiu com diagnóstico diferencial de hanseníase MB e tuberculose.

\section{RELATO DE CASO}

Paciente, sexo masculino, 68 anos, morador de rua, admitido no PS do Hospital Municipal da cidade de Paracatu -MG no dia 01/03/2016. Levado por terceiros que relataram tê-lo encontrado torporoso, apresentando lesão ulcerada, secretiva, em 3o pododáctilo direito (PDD). O paciente relatou ser diabético, hipertenso e portador de quadro de anemia crônica, em tratamento irregular. Nega etilismo e tabagismo.

Ao exame físico apresentou-se desidratado (++/++++), hipocorado (++/++++), Glasgow 13/15, pupilas fotorreagentes, frequência cardíaca: 64 batimentos por minuto, pressão arterial: 130x90 mmHg, Glicemia capilar: $417 \mathrm{mg} / \mathrm{dL}$, Aparelho respiratório: murmúrios vesiculares fisiológicos sem ruídos adventícios, Aparelho cardiovascular: ritmo cardíaco regular em dois tempos, bulhas normofonéticas e normorítmicas com ausência de sopros audíveis. Rotina laboratorial: Hemácias: 2,61 milhões/mm³, Hemoglobina: 8,0 g/dL; Hematócrito: 25,0\%; Leucócitos: $6.600 \mathrm{~mm}^{3}$; Linfócitos: $1848 \mathrm{~mm}^{3}$. 
No dia 02/03/2016, o paciente foi admitido na clínica médica do Hospital Municipal de Paracatu - MG. Teve sua primeira evolução pela manhã, na qual relatou tosse não produtiva há 3 semanas associada a febre não aferida e emagrecimento. Ao exame físico em MMII (membros inferiores), presença de lesão ulcerada em pé direito e diversas escoriações.

No segundo dia de internação hospitalar $(\mathrm{IH})$, apresentou resultado de elementos e sedimentos anormais da urina (EAS): glicose: +++ (1000 mg/dL); leucócitos: campos repletos; cilíndros: granulosos. Início da prescrição médica de ceftriaxona, também, solicitado interconsulta com a cirurgia vascular devido a lesão ulcerativa. Ao exame: úlcera em extremidade de $3^{\circ}$ PDD com tecidos necróticos e viabilidade tecidual circunjacente. Hipótese diagnóstica: pé diabético; Conduta: manter antibiótico e controle glicêmico rigoroso.

No quarto dia de evolução, em MMII: pulsos presentes, panturrilhas livres, sem edema, lesões em cicatrização unilateral, lesão ulcerada única em $3^{\circ}$ PDD. Após cinco dias de IH relatou ainda, dor em MMII do tipo queimação associada a parestesia, ao exame presença de lesões descamativas e crostosas, lesão ulcerada com odor fétido e secreção purosanguinolenta em 3ำ PDD, ausência de edema e empastamento. Sob dúvida diagnóstica devido apresentação das lesões em MMII, foi solicitado avaliação com especialista em hanseníase. Novamente realizada interconsulta vascular, na qual: paciente apresentando área de necrose em 3PDD, bem delimitada e sem sinais infecciosos adjacentes.

Realizada avaliação do serviço de hanseníase, no décimo dia de $\mathrm{IH}$. O paciente apresentava espessamento cubital direito, afilamento de músculos interósseos em mãos, neurite ulnar e múltiplas necroses em MMII (características venosas), neuropatia periférica crônica, pé caído + mão em garra + mal perfurante plantar.

No décimo quarto dia iniciada poliquimioterapia e preenchimento de notificação para Hanseníase. No vigésimo dia de $\mathrm{IH}$, foi realizado procedimento pela cirurgia vascular, desbridamento de 3 PDD distalmente. Ato sem intercorrências e realizado sob anestesia local.

No dia 14/04/2016, ao exame físico de MMII: panturrilhas livres, ausência de edema, plegia em pododáctilos, ausência de sensibilidade. Foi solicitada interconsulta vascular devido apresentação de sinais de isquemia e drenagem de secreção purulenta de odor fétido em $3^{\circ}$ PDD. A cirurgia vascular declarou infecção em artelho com inviabilidade do mesmo, sendo encaminhado para amputação do artelho, com autorização do paciente. Início de Imipinem e Vancomicina até cultura. Foi realizada a amputação de $3^{\circ}$ PDD.

Foi realizada troca de medicação no dia 18/04/2016 visto resultado de cultura, evidenciada Escherichia coli. Ajustado prescrição para Piperacilina + Tazobactam.

Aos dias 24 de abril de 2016, as coletas cessaram, estando ainda o paciente S.A.S. internado no Hospital Municipal para continuidade do tratamento e suporte clínico, com diagnóstico de Hipertensão Arterial Sistêmica, Diabetes Mellitus II e Hanseníase. 


\section{DISCUSSÃO}

O diabetes mellitus constitui um dos principais fatores de risco para doenças do aparelho circulatório. Entre suas consequências mais frequentes, encontra-se o infarto agudo do miocárdio, o acidente vascular encefálico, a insuficiência renal crônica e as amputações de pés e pernas. As neuropatias que levam ás amputações têm a prevalência elevada no primeiro período (0-5 anos) da doença e se instalam em $40 \%$ dos diabéticos, após 15 anos, estando a doença vascular periférica presente em $45 \%$ dos diabéticos com mais de 20 anos de doença, sendo estimado que $15 \%$ dos pacientes desenvolverão úlceras nos membros inferiores, gangrenas e amputações (GRILLO MFF e GORINI MIPC, 2007).

No caso clínico apresentado, o paciente foi admitido para tratamento de lesão ulcerativa em 3o pododáctilo, diagnosticada diante da clinica como uma consequência da neuropatia diabética, formando lesão característica, sem demais associações a novas doenças. Foram também observadas lesões crostosas e descamativas em membros inferiores que não foram tratadas incialmente, uma vez vistas como hipótese diagnostica de má cicatrização de feridas em membros devido doença circulatória instalada. Foram utilizados para tratamento: conduta terapêutica de desbridamento da ferida em 3o pododáctilo e medicamentosa.

Entre as polineuropatias tem-se: as neuropatias associadas ao alcoolismo e carenciais, neuropatia diabética, as neuropatias sensitivas criptogênicas e outras hereditárias sensitivo-motoras, com espessamento de nervos, a neuropatia do hipotireoidismo e Infecção pelo HIV. As polineuropatias sensitivas ou sensitivas e autonômicas adquiridas também devem estar elencadas neste grupo de síndromes neurológicas cuja causa pode ser a hanseníase (GARBINO JA, 2007).

A permanência das lesões indolores com diminuição de sensibilidade em membros inferiores, levou a suspeita e investigação do diagnóstico diferencial de hanseníase, não investigada anteriormente por associação dos sintomas com o diabetes mellitus. Essa associação foi consistente pelo fato de uma das complicações diabéticas ser a perda da sensibilidade, característica das lesões encontradas, e também condizente com a hanseníase. Contudo, a maior diferença entre as patologias apresentadas é que 0 surgimento de lesões indolores é característico da hanseníase, enquanto o diabetes oculta algumas lesões devido a perda da sensibilidade pela neuropatia (SOUZA CFD, et al., 2010).

As características específicas da evolução do paciente foram decisivas para o diagnóstico de hanseníase efetuado pelo serviço especializado. Esse diagnóstico se baseou única e exclusivamente no quadro clinico do paciente, que apresentava espessamento cubital direito, afilamento de músculos interósseos das mãos, neurite ulnar, múltiplas necroses em membros inferiores, pé caído, mãos em garra e mal perfurante plantar.

Conforme preconizado na conduta protocolada para tratamento da hanseníase foi iniciada poliquimioterapia (PQT). Além do tratamento medicamentoso, foi solicitada e realizada amputação de $3^{\circ}$ PDD, devido á processo infeccioso disseminado refratário a antibióticoterapia (PACE AE, et al., 2003). 


\section{CONSIDERAÇÕES FINAIS}

A partir das análises realizadas no presente estudo, a neuropatia diabética é uma das complicações comuns do diabetes mellitus tipo 2 , e associada a diagnósticos diferenciais como a hanseníase, pode mascarar seus sinais e sintomas, causando prejuízo ao prognóstico do paciente. Isso se deve ao fato de ambas apresentarem diminuição da sensibilidade, entretanto a hanseníase leva ao surgimento de lesões indolores, enquanto a neuropatia diabética mascara lesões de característica indolor. No relato apresentado, a gravidade do quadro de pé diabético acabou sobrepondo a atenção médica quanto as lesões indolores de MMII, que caracterizavam parte do quadro clínico de hanseníase descoberto posteriormente, isso se deu pelo fato de as lesões presentes características do quadro de hanseníase se assemelharem, quanto a diminuição de sensibilidade, a lesões provenientes da neuropatia diabética. Evidencia-se então, a necessidade de observação médica aos mínimos sinais e sintomas, e associação dos mesmos com quadros de diagnósticos diferenciais.

\section{REFERÊNCIAS}

1. BORTOLETTO MSS, HADADD MCL, KARNO ME et al. Pé diabético, uma avaliação sistematizada. Arq. Ciênc. Saúde Unipar, Umuarama, 2009; 13(1): 37-43.

2. GAMBA MA, GOTLIEB SLD, BERGAMASCHI DP, VIANNA LAC et al. Amputações de extremidades inferiores por diabetes mellitus: estudo caso-controle. Revista Saúde Pública, 2004; 38(3): pp. 399-404.

3. GARBINO, José Antônio. O paciente com suspeita de hanseníase primariamente neural. Hansenologia Internationalis (Online), 2007, 2007.

4. GRILLO, Maria de Fátima Ferreira; GORINI, Maria Isabel Pinto Coelho. Caracterização de pessoas com diabetes mellitus tipo 2. Revista brasileira de enfermagem. Brasília, 2007; 60(1): pp. 49-54.

5. GROSSI MA. Hanseníase no Brasil. Revista da Sociedade Brasileira de Medicina Tropical, 2003; 36(3): pp.373382.

6. GROSS JL, SILVERIO S, CAMARGO JL et al. Diabetes Melito: Diagnóstico, Classificação e Avaliação do Controle Glicêmico. Arq Bras Endocrionol Metabol, 2002; 46(1), São Paulo.

7. LASTORIA JC, PUTINATTI MSMA, DIÓRIO SM et al. Índices baciloscópico e morfológicos na hanseníase após doze doses do esquema poliquimioterápico (PQT/OMS). Hansen Int, 2006, 31(1): pp.15-21.

8. OLIVEIRA JEPR. Diretrizes da Sociedade Brasileira de Diabetes 2017-2018. -- São Paulo: Editora Clannad, 2017.

9. PACE AE, NUNES PD, VIGO KO et al. O conhecimento dos familiares acerca da problemática do portador de diabetes mellitus. Revista Latino-Am Enfermagem, 2003; 11(3): pp. 312-319.

10. PEREIRA HLA, RIBEIRO SLE, CICONELLI RM et al. Avaliação por imagem do comprometimento osteoarticular e de envolvimento de nervos periféricos na hanseníase. Revista Brasileira de Reumatologia, 2006; 46(1): pp.30-35.

11. SOUZA CFD, SLAIBI EB, PEREIRA RN et al. A importância do diagnóstico de hanseníase na prevenção de incapacidades. Hansenol. 2010; Int. (Online) 35(2). 